\title{
Chronic hyperaldosteronism in Cryptochrome-null mice induces high-salt- and blood pressure- independent kidney damage in mice
}

\author{
Dwi Aris Agung Nugrahaningsih ${ }^{1}$, Noriaki Emoto ${ }^{1,2}$, Nicolas Vignon-Zellweger ${ }^{2}$, Eko Purnomo ${ }^{1}$, \\ Keiko Yagi ${ }^{2}$, Kazuhiko Nakayama ${ }^{1,2}$, Masao Doi ${ }^{3}$, Hitoshi Okamura ${ }^{3}$ and Ken-ichi Hirata ${ }^{1}$
}

\begin{abstract}
Although aldosterone has an essential role in controlling electrolyte and body fluid homeostasis, aldosterone also exerts certain pathological effects on the kidney. Several previous studies have attempted to examine these deleterious effects. However, the majority of these studies were performed using various injury models, including high-salt treatment and/or mineralocorticoid administration, by which the kidney changes observed were not only due to aldosterone but also due to prior injury caused by salt and hypertension. In the present study, we investigated aldosterone's pathological effect on the kidney using a mouse model with a high level of endogenous aldosterone. We used cryptochrome-null (Cry 1, 2 DKO) mice characterized by high aldosterone levels and low plasma renin activity and observed that even under normal salt exposure conditions, these mice showed increased albumin excretion and kidney tubular injury, decreased nephrin expression and increased reactive oxygen species production in the absence of hypertension. Exposure to high salt levels exacerbated the kidney damage observed in these mice. Moreover, we noted that decreasing blood pressure without blocking aldosterone action did not provide beneficial effects to the kidney in high-salt-treated Cry 1, 2 DKO mice. Thus, our findings support the hypothesis that aldosterone has deleterious effects on the kidney independent of high-salt exposure and high blood pressure.
\end{abstract} Hypertension Research (2014) 37, 202-209; doi:10.1038/hr.2013.143; published online 10 October 2013

Keywords: aldosterone; blood pressure; kidney; salt

\section{INTRODUCTION}

Aldosterone is a mineralocorticoid hormone produced in the cortex of the adrenal glands. Under physiological conditions, aldosterone's binding with specific intracellular mineralocorticoid receptors (MRs) in the distal nephron regulates electrolyte and water homeostasis. ${ }^{1}$ Thus, aldosterone regulates blood volume and blood pressure. ${ }^{2}$ However, aldosterone also contributes to the development of kidney damage, as reported in several studies. ${ }^{3,4}$ Primary aldosteronism, characterized by high plasma aldosterone levels and low renin activity, is often correlated with an increased risk of kidney damage. ${ }^{5}$

Remnant kidney models in animals with elevated plasma aldosterone levels showed hypertension and proteinuria. ${ }^{6}$ Another study with increased mineralocorticoid levels following deoxycorticosterone acetate administration in rats subjected to unilateral nephrectomy found that high-salt treatment induced hypertension, peritubular capillary loss and tubular interstitial fibrosis. ${ }^{7}$ Moreover, in rats treated with salt and aldosterone for 28 days, severe kidney damage and hypertension were observed. ${ }^{8}$ Using these models, hypertension's role in the development of kidney damage could not be ruled out.
Studies on several animal kidney injury models have indicated that MR blocker administration can ameliorate kidney damage independent of blood pressure. ${ }^{9,10}$ To further elucidate aldosterone's effect on the kidney independent of blood pressure, some researchers have decreased blood pressure without interfering with aldosterone using hydralazine treatment, ${ }^{11}$ whereas other researchers have administered a non-hypotensive dose of an MR blocker ${ }^{12}$ or lowdose aldosterone. ${ }^{13}$ Although many aldosterone-induced renal pathology studies have been performed, most of these studies used injury models, such as subtotal renal ablation and unilateral nephrectomy, mineralocorticoid administration, high-salt treatment or a combination of these methods, by which aldosterone's pathological effects on the kidney cannot be clearly distinguished from previously existing injuries caused by salt and hypertension.

To study aldosterone-induced kidney damage in the absence of prior kidney manipulation, high-salt treatment and/or mineralocorticoid administration, we used Cry 1, 2 DKO mice. Cryptochrome is known to regulate the circadian rhythm. ${ }^{14}$ Cry 1, 2 DKO mice show increased mRNA expression and protein levels of $3 \beta$ hydroxysteroid

${ }^{1}$ Division of Cardiovascular Medicine, Department of Internal Medicine, Kobe University Graduate School of Medicine, Kobe, Japan; ${ }^{2}$ Department of Clinical Pharmacy, Kobe Pharmaceutical University, Kobe, Japan and ${ }^{3}$ Department of System Biology, Graduate School of Pharmaceutical Sciences, Kyoto University, Kyoto, Japan

Correspondence: Professor N Emoto, Division of Cardiovascular Medicine, Department of Internal Medicine, Kobe University Graduate School of Medicine, 7-5-1, Kusunoki-cho, Chuo-ku, Kobe 650-0017, Japan.

E-mail: emoto@med.kobe-u.ac.jp

Received 18 June 2013; revised 31 July 2013; accepted 2 August 2013; published online 10 October 2013 
dehydrogenase, the enzyme involved in aldosterone synthesis. The enzyme is expressed particularly in the zona glomerulosa, where aldosterone production is known to exclusively take place. Thus, Cry 1, 2 DKO mice present with high aldosterone levels but low plasma renin activity. ${ }^{15}$

\section{METHODS}

\section{Animal experiments}

In the present study, we used cryptochrome-null (Cry 1, 2 DKO) mice, which characteristically show high plasma aldosterone levels and suppressed renin activity. ${ }^{15}$ The development of Cry 1,2 DKO mice has been described previously. ${ }^{16}$ During the course of the study, all the mice were maintained at a stable environmental temperature with a 12-h light and dark cycle and had free access to water and chow. All the experimental protocols were performed based on the animal experiment guidelines of Kobe University, Kobe, Japan.

We used Cry 1, 2 DKO mice and wild-type (WT) littermates as controls. We divided 12-week-old male mice into the following six groups: WT mice receiving normal salt treatment (WTNS; $n=17$ ), WT mice receiving high-salt treatment (WTHS; $n=16$ ), Cry 1, 2 DKO mice receiving normal salt treatment (CRYNS; $n=13$ ), Cry 1, 2 DKO mice receiving high-salt treatment (CRYHS; $n=20$ ), Cry 1, 2 DKO mice receiving high-salt and spironolactone treatment (CRYSPI; $n=13$ ) and Cry 1, 2 DKO mice receiving high-salt and hydralazine treatment (CRYHYD; $n=6$ ). All the mice underwent the respective treatments for 32 weeks. The normal salt chow had a $0.2 \% \mathrm{NaCl}$ content, whereas the high-salt chow had a $3.15 \% \mathrm{NaCl}$ content, and the drinking water contained $1 \% \mathrm{NaCl}$ and $0.2 \% \mathrm{KCl}$. Spironolactone or hydralazine was administered in the drinking water. The doses of spironolactone (Sigma-Aldrich, St Louis, MO, USA) and hydralazine (Sigma-Aldrich) were $6 \mathrm{mg} \mathrm{kg}^{-1}$ body weight per day and $25 \mathrm{mg} \mathrm{kg}^{-1}$ body weight per day, respectively. Spironolactone was dissolved in ethanol and then diluted in drinking water.

\section{Blood pressure measurement}

Blood pressure was determined in conscious, trained mice using a non-invasive computer-automated tail-cuff system (BP-98A, Softron, Tokyo, Japan). The average value of 10 measurements was used for data analysis.

\section{Blood and urine biochemical analysis}

After 32 weeks of treatment, blood was obtained for biochemical analysis by cardiac puncture before the mice were killed. A 24-hurine collection was performed using metabolic cages 1 day before the mice were killed. Creatinine concentrations in the serum and urine were measured using an enzymatic assay (Nescoat VLII CRE Kit; Alfresa Pharma Corp, Osaka, Japan). A commercially available enzyme-linked immunosorbent assay kit was used to measure urine albumin (Bethyl Laboratories, Montgomery, TX, USA) according to the manufacturer's instructions. Serum aldosterone was measured using a commercially available enzyme immunoassay kit (Enzo Life Sciences, Plymouth Meeting, PA, USA) according to the protocol described by the manufacturer. Serum and urine electrolytes were measured using an ionselective electrode and calculated using the Nernst equation.

\section{Gene expression}

The total RNAs from the removed kidney were extracted using RNAIsoPlus (TaKaRa, Dalian, China). Reverse transcription was performed using ReverTra Ace (Toyobo, Osaka, Japan) according to the manufacturer's instructions. The quantification of renin, MR, $11 \beta$ hydroxysteroid dehydrogenase type 2 (11BHSD2), nephrin and glucose-6-phosphate dehydrogenase (G6PD) gene expression levels was performed using the Thunderbird SYBR qPCR Mix (Toyobo, Osaka, Japan) according to the protocol described by the manufacturer. The primers used in this study were as follows: renin (forward: 5'-ATGAAGGGGGTGTCTGTGGGGTC-3'; reverse: $5^{\prime}$-ATGTCGGGGAGGGT GGGCACCTG- $3^{\prime}$ ), MR (forward: $5^{\prime}$-GGAAACCAAAGGCTACCACA-3'; reverse: $5^{\prime}$-AGTGTGGAGGACCTGTGACC-3'), 11BHSD2 (forward: 5'-TTTG GTGCACTTGAGCTGAC-3'; reverse: $5^{\prime}$-GGTATGGCATGTCTCCTGCT-3'), nephrin (forward: 5'-ACTACGCCCTCTTCAAATGCA-3'; reverse: $5^{\prime}$-TCGAG GGCCTCATACCTGAT-3'), G6PD (forward: 5'-GTTAAATGGGCCAGCG
AAG-3'; reverse: $5^{\prime}$-CCTGCTCTGCCATGATGTTT- $3^{\prime}$ ) and glyceraldehyde 3-phosphate dehydrogenase (forward: $5^{\prime}$-TGTGTCCGTCGTGGATCTGA-3'; reverse: $5^{\prime}$-TTGCTGTTGAAGTCGCAGGAG-3').

\section{Histopathology quantification}

The kidneys of the mice were obtained and fixed with $4 \%$ paraformaldehyde overnight at room temperature before being processed in paraffin. Thereafter, $4-\mu \mathrm{m}$ sectioned samples were used for periodic acid-Schiff (PAS) staining and Sirius red staining. Tubular injury was assessed at $\times 200$ magnification in samples from each group that were stained with PAS. Tubular injury was assessed using 15 non-overlapping fields of view. Tubular injury was defined as the presence of tubular epithelial swelling, tubular epithelial vacuolization, tubular necrosis and loss of the brush border and was categorized into five grades from 0 to 4 , as described previously. ${ }^{17}$ Glomerulosclerosis index quantification was performed using 20 PAS-stained glomeruli. The glomeruli were scored on a 0 to 4 scale for glomerulosclerosis, as described previously. ${ }^{18}$ The scores for all glomeruli were averaged and presented as the glomerulosclerosis index. Tubular interstitial fibrosis quantification was performed using 20 Sirius red-stained sections at $\times 200$ magnification. The fibrosis area was quantified using ImageJ software (US National Institutes of Health, Bethesda, MD, USA) and presented as the percentage area of fibrosis. Nephrin staining was performed on cryosections. Briefly, sectioned slides were incubated with an antibody against nephrin (Progen Biotechnik GmbH, Heidelberg, Germany) at $100 \times$ dilution overnight at $4{ }^{\circ} \mathrm{C}$. Nephrin expression was reported as the percentage of the entire glomerulus area that was positive for nephrin. Fifteen glomeruli were assessed for nephrin expression using the Image software.

\section{Assessment of reactive oxygen species production}

The reactive oxygen species (ROS) production assessment was performed on cryosections stained with dihydroethidium. Briefly, cryosections were incubated with $5 \mu \mathrm{m}$ dihydroethidium in dimethyl sulfoxide at $37^{\circ} \mathrm{C}$ for $30 \mathrm{~min}$. Dihydroethidium intensity quantification was performed using 15 visual fields at $\times 200$ magnification.

\section{Statistical analyses}

All data are presented as the mean \pm s.e.m. We assumed that the groups were not normally distributed. Therefore, we performed statistical analysis of multiple groups using the non-parametric Kruskal-Wallis test, followed by the Mann-Whitney $U$-test between two groups. A $P$-value of $<0.05$ was considered significant.

\section{RESULTS}

Serum aldosterone and renin gene expression levels

Serum aldosterone levels were significantly greater in Cry 1, 2 DKO mice compared with WT mice. High levels of serum aldosterone in Cry 1, 2 DKO mice were persistent even under high-salt treatment, whereas serum aldosterone levels in WT mice were significantly decreased following high-salt treatment (Figure 1a). To assess whether aldosterone production is independent of renin gene expression, we examined renin gene expression in the whole kidney of Cry 1, 2 DKO and WT mice. We noted that renin gene expression in the whole kidney was similar among the groups (Figure 1b). The aldosterone receptor (MR) expression in the whole kidney was also similar among the groups. Because MRs are nonspecific receptors and their specificity is determined by $11 \mathrm{BHSD} 2,{ }^{19}$ we measured $11 \mathrm{BHSD} 2$ expression in the whole kidney as well. However, we did not note any difference in 11BHSD2 expression among the different groups (Figures $1 \mathrm{c}$ and $\mathrm{d}$ ).

\section{Blood pressure examination}

Blood pressure was similar between the CRYNS and WTNS. The systolic blood pressure in the CRYHS increased significantly. Moreover, spironolactone treatment did not decrease the blood pressure in 

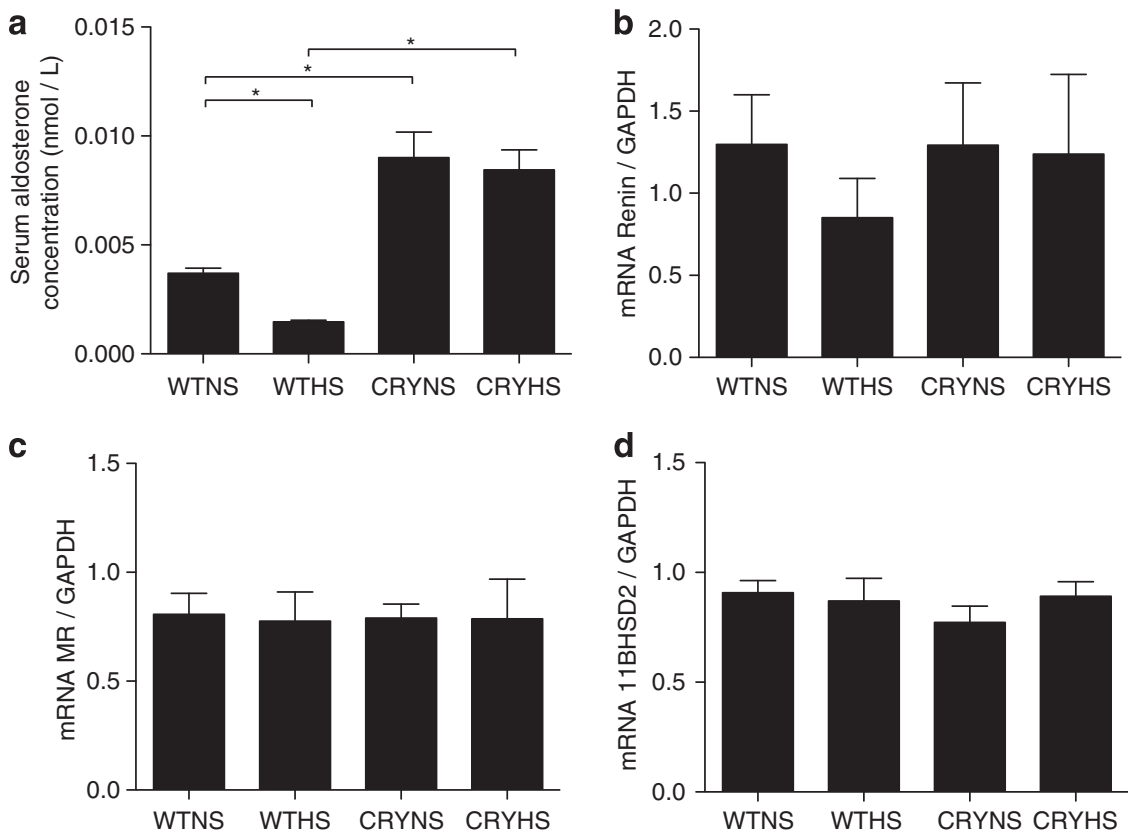

Figure 1 Serum aldosterone concentration (a) and renin (b), mineralocorticoid receptor (MR) (c) and 11 beta hydroxysteroid dehydrogenase type 2 (11BHSD2) (d) mRNA expression. Serum aldosterone concentration measurement was performed in five to nine mice. (b-d) The graphs represent the ratio between mRNA expression and the expression of glyceraldehyde 3-phosphate dehydrogenase (GAPDH) mRNA in the whole kidney ( $n=4$ ). Data are presented as the mean \pm s.e.m. ${ }^{*} P<0.05$ using the Mann-Whitney $U$-test.

the CRYSPI. However, hydralazine treatment significantly decreased the blood pressure in the CRYHYD (Figure 2).

\section{Blood and urine biochemical analysis}

Creatinine clearance levels were similar between Cry 1, 2 DKO and WT mice receiving normal salt treatment (Figure 3a). High-salt treatment increased the creatinine clearance in Cry 1, 2 DKO mice but not in WT mice. Spironolactone or hydralazine treatment in high-salt-treated Cry 1, 2 DKO mice did not decrease creatinine clearance. To further examine the effect of hyperaldosteronism on kidney function, we assessed the urinary albumin levels. Under normal salt treatment, urinary albumin levels were significantly higher in Cry 1, 2 DKO mice compared with those of WT mice (Figure 3b). High-salt treatment in Cry 1, 2 DKO mice further increased urinary albumin excretion. However, treatment with spironolactone, but not hydralazine, decreased urinary albumin excretion. Sodium and potassium concentrations both in serum and urine were the same between Cry 1, 2 DKO and WT mice under the same treatment. Treatment with high salt and spironolactone or hydralazine in Cry 1, 2 DKO and WT mice induced no statistically significant difference in serum sodium or potassium concentrations. High-salt treatment increased the urine sodium and potassium concentrations both in Cry 1, 2 DKO and WT mice. Treatment with high salt and spironolactone or hydralazine in Cry 1,2 DKO mice also resulted in increased urine sodium and potassium concentrations (Figures 3c-f).

\section{Kidney histology assessment}

Kidney tubular injury and kidney fibrosis were measured in the tubular area of the kidney by PAS staining and Sirius red staining, respectively (Figure 4a). Glomerulosclerosis was measured in the glomerular area of the kidney using PAS staining (Figure 4a). Kidney tubular injury was observed in the CRYNS group but not in the

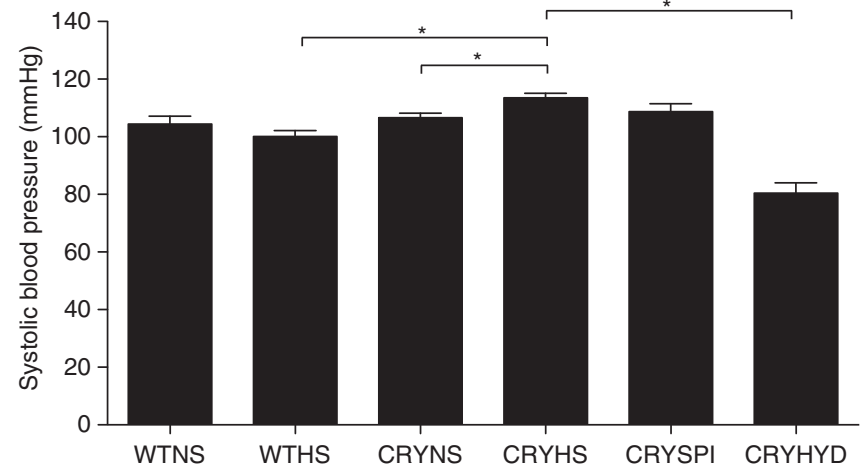

Figure 2 Systolic blood pressure measured in conscious mice using the tailcuff method. Data are presented as the mean \pm s.e.m. $n=6-14$. ${ }^{*} P<0.05$ using the Mann-Whitney U-test.

WTNS group (Figure $4 \mathrm{~b}$ ). The mice in the CRYHS group exhibited more severe kidney tubular injury compared with mice in the WTHS and the CRYNS groups. Moreover, spironolactone treatment ameliorated kidney tubular injury in the CRYSPI group, whereas hydralazine treatment did not improve kidney tubular injury in the CRYHYD group. The quantifications of glomerulosclerosis and tubular interstitial fibrosis were similar among the treatment groups (Figures $4 \mathrm{c}$, d). Nephrin expression in the glomerular area was analyzed by immunostaining (Figure 5a). Mice in the CRYNS group showed lower levels of nephrin expression compared with those in the WTNS group (Figures $5 \mathrm{~b}$ and $\mathrm{c}$ ). The level of nephrin expression in the CRYHS group was lower compared with that of the WTHS and CRYNS groups. However, treatment with spironolactone, but not hydralazine, improved the nephrin expression levels in high-salttreated Cry 1, 2 DKO mice. 


\section{a}
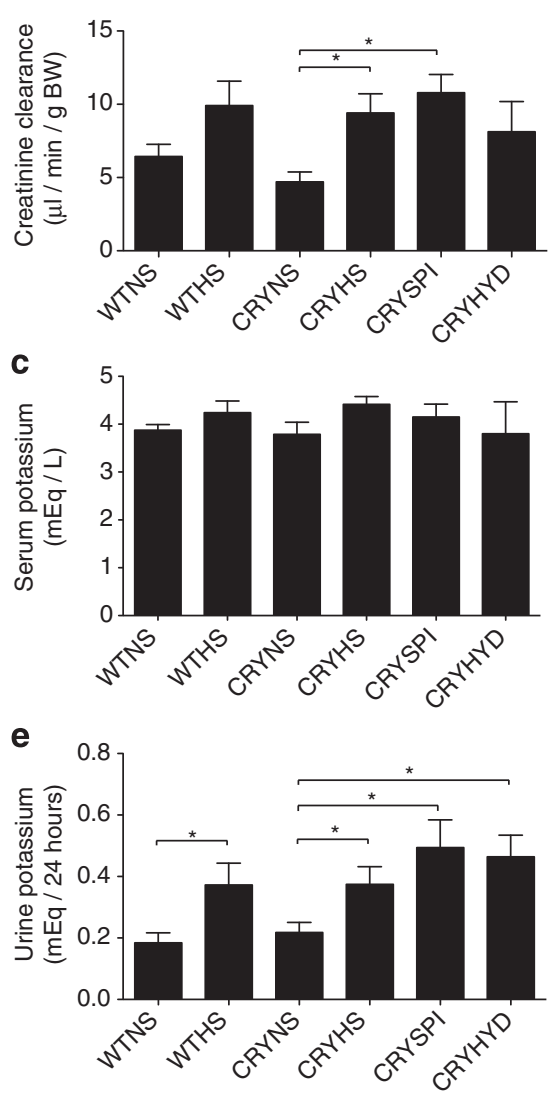

b

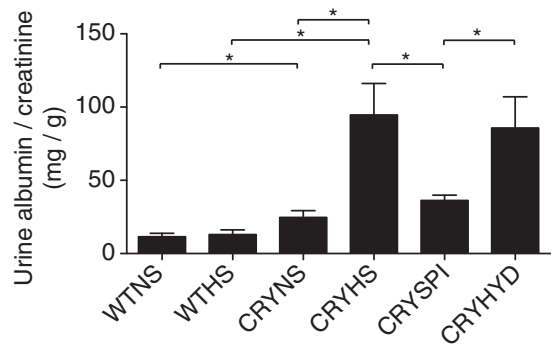

d

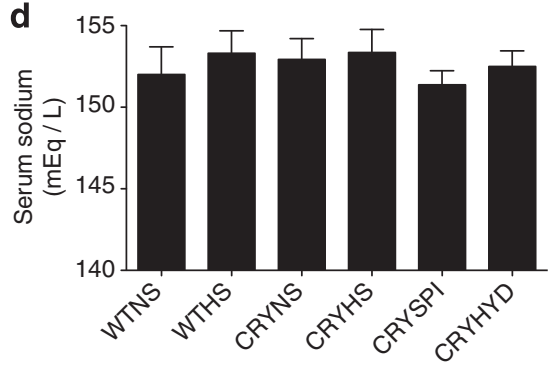

f

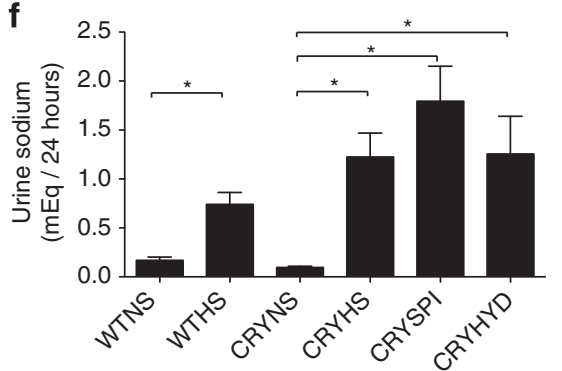

Figure 3 Creatinine clearance (a), albuminuria (b), serum potassium level (c), serum sodium level (d), urine potassium level (e) and urine sodium level (f) of mice after 32-week treatment. Data are presented as the mean \pm s.e.m. $n=6-13$. ${ }^{*} P<0.05$ using the Mann-Whitney $U$-test.

\section{ROS production}

ROS production in the kidney was examined using dihydroethidium staining, as depicted in Figure 6a. We observed significantly higher levels of ROS in the CRYNS group compared with the WTNS group. Mice in the CRYHS group showed a further increase in ROS production in the kidney. Spironolactone treatment in high-salttreated Cry 1, 2 DKO mice caused a decrease in ROS production, whereas hydralazine treatment did not cause such a decrease (Figure 6b). To further examine the cause of increased ROS production, we examined the expression of G6PD. The G6PD expression levels decreased in the CRYNS and CRYHS groups compared with those of the WTNS and WTHS groups, respectively. Treatment with spironolactone, but not hydralazine, improved the G6PD expression levels in high-salt-treated Cry 1,2 DKO mice (Figure 6c).

\section{DISCUSSION}

In the present study, we demonstrated chronic hyperaldosteronism's pathological effects on the kidney independent of high-salt treatment and hypertension in Cry 1, 2 DKO mice. Our model showed that even under normal salt treatment, chronic hyperaldosteronism resulted in albuminuria, tubular injury, decreased nephrin expression and increased ROS production in the absence of hypertension. Notably, the pathological processes due to hyperaldosteronism took place after a long follow-up period ( 32 weeks). We further confirmed that high-salt exposure in addition to the previous condition induced more severe kidney damage compared with that observed in hyperaldosteronism cases alone. Finally, we also demonstrated that kidney damage induced by hyperaldosteronism and high-salt exposure is independent of blood pressure.

Despite high plasma aldosterone levels, the Cry 1, 2 DKO mice under normal salt treatment showed no differences in the blood pressure or serum potassium level compared with those of WT mice under the same treatment. Although hyperaldosteronism is frequently associated with hypertension and hypokalemia, normotensive or normokalemic patients with hyperaldosteronism have also been reported. ${ }^{20-22}$ Therefore, hypertension should not be used as a prerequisite for a primary aldosteronism diagnosis. ${ }^{20}$ It is also suggested that normokalemia should not exclude the possibility of primary hyperaldosteronism..$^{23}$

We noted that the Cry 1, 2 DKO mice in the present study developed albuminuria following normal salt treatment and in the absence of elevated blood pressure. Aldosterone exerts its effect by binding with MRs. In the kidney, MRs are mainly expressed in the distal tubules and collecting ducts. ${ }^{24}$ Lower MR expression has been reported in glomeruli, particularly in mesangial cells ${ }^{25}$ and podocytes ${ }^{26}$ in which aldosterone may act directly. In unilateral nephrectomized rats receiving aldosterone and high-salt treatment, proteinuria was found to be associated with a decrease in the levels of nephrin, which is a podocyte-related protein. ${ }^{26}$ Nephrin is expressed specifically in the slit diaphragm of podocytes and therefore has an important role in the function of the glomerular filtration barrier. ${ }^{27}$ In the present study, we observed that Cry 1,2 DKO mice showed a decrease in nephrin expression levels. Therefore, we suggest that 
a
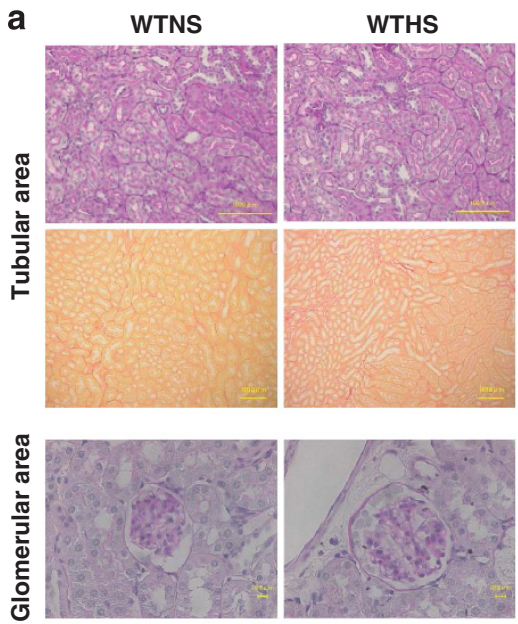

b

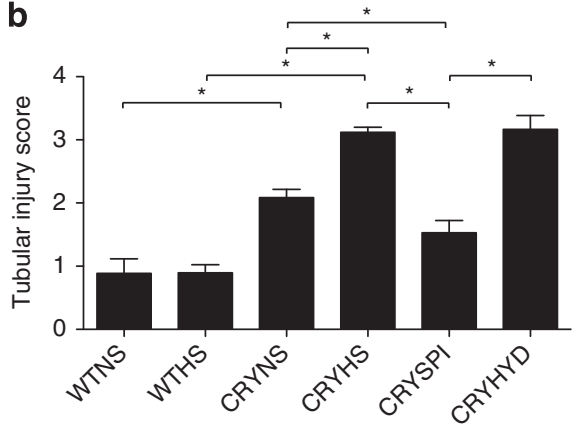

CRYNS
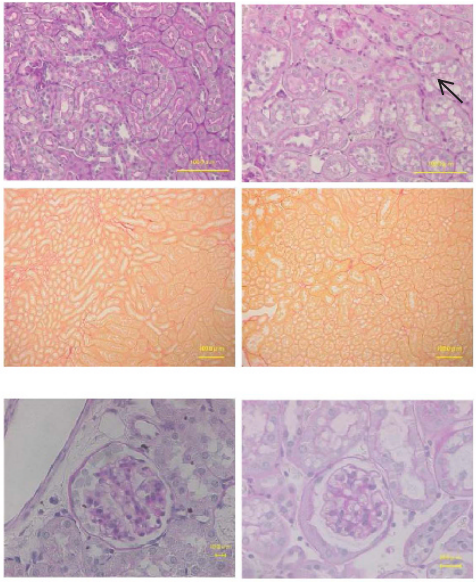

CRYHS
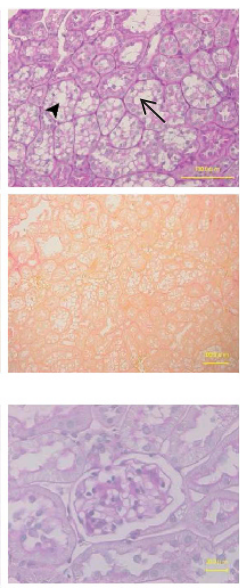

C
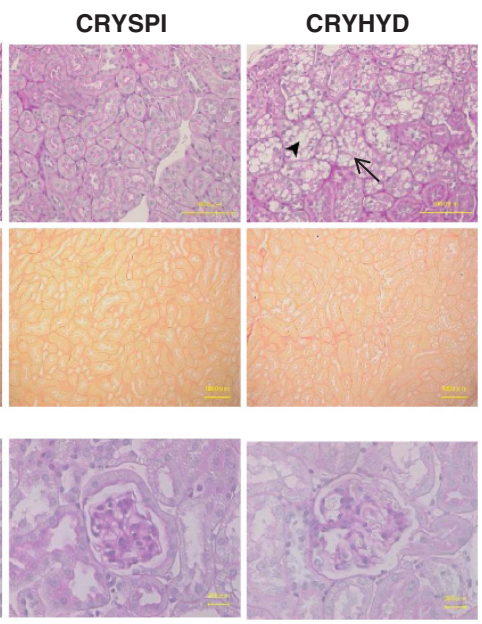

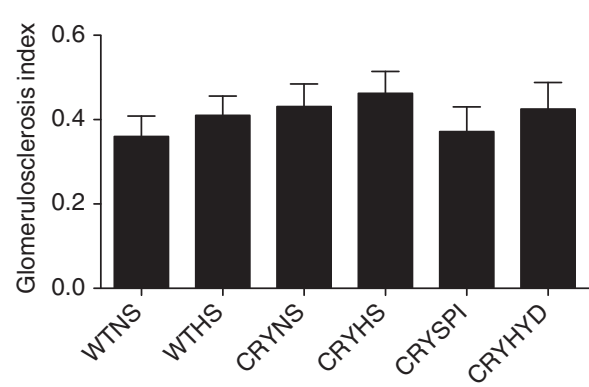

d

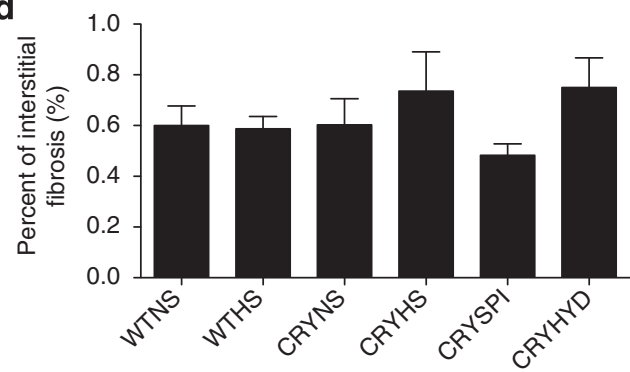

Figure 4 Representative pictures of periodic acid-Schiff (PAS) staining of the tubular area, Sirius red staining of the tubular area and PAS staining of the glomerular area (a). The graphs represent the quantification of the tubular injury score (b), glomerulosclerosis (c) and tubular interstitial fibrosis (d). Tubular injury was identified by PAS staining of the tubular area as tubular vacuolization (arrow) and the loss of brush border (arrowhead). Data are presented as the mean \pm s.e.m. $n=6-14 .{ }^{*} P<0.05$ using the Mann-Whitney U-test.

a decrease in nephrin expression in Cry 1,2 DKO mice results in disruption of the glomerular filtration barrier and thus influences, at least in part, the urinary albumin level.

In our study, Cry 1, 2 DKO mice characterized by hyperaldosteronism showed increased kidney ROS production in the absence of high-salt exposure, which is consistent with the findings of previous studies. ${ }^{28,29}$ Administration of the ROS detoxifier agent tempol to uninephrectomized rats receiving high-salt treatment and aldosterone has been reported to ameliorate kidney damage, ${ }^{11}$ indicating that ROS production might be one of the factors responsible for aldosteroneinduced kidney damage. The present study results suggest that ROS production applies to cases with only hyperaldosteronism as well. In addition, we noted that G6PD expression decreased in Cry 1, 2 DKO mice. G6PD is the first enzyme in the pentose phosphate pathway, which is an important source of NADPH (Nicotinamide Adenine Dinucleotide Phosphate). ${ }^{29}$ A previous study involving aldosterone- and salt-treated mice indicated a decrease in G6PD expression and activity in the aorta; this decrease promotes increased ROS accumulation due to decreased production of NADPH, which is an important intracellular reducing equivalent. ${ }^{30}$ G6PD deficiency in mice also reportedly results in increased renal oxidative stress and urinary albumin levels. ${ }^{29}$ Thus, the decrease in G6PD expression levels in Cry 1, 2 DKO mice may have a role in increased ROS production and urinary albumin levels.

Compared with animal models combining unilateral nephrectomy with high-salt and mineralocorticoid exposure, wherein a relatively short-term treatment (4-8 weeks) produced severe kidney damage including glomerulosclerosis and kidney fibrosis, ${ }^{8,12}$ the kidney damage observed in the normal salt-treated Cry 1, 2 DKO mice over 32 weeks was less severe. Moreover, even after 32 weeks of high-salt treatment, the Cry 1, 2 DKO mice did not develop glomerulosclerosis or interstitial fibrosis. On the basis of these 

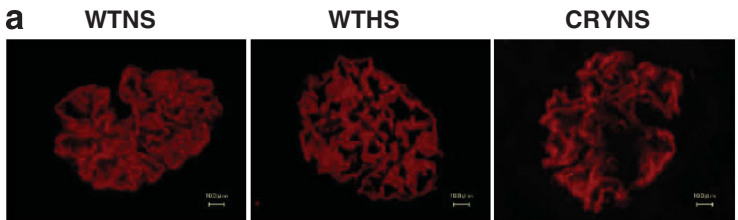

b

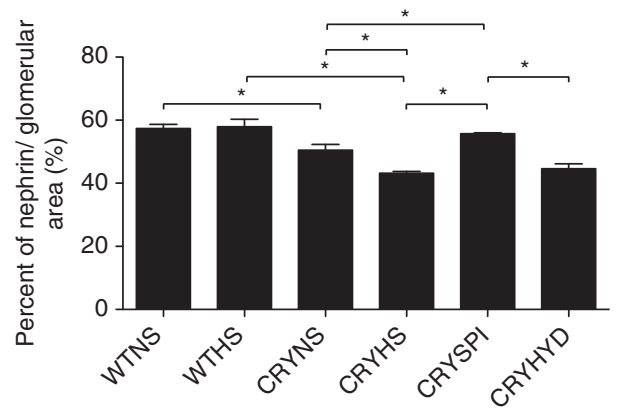

CRYHS
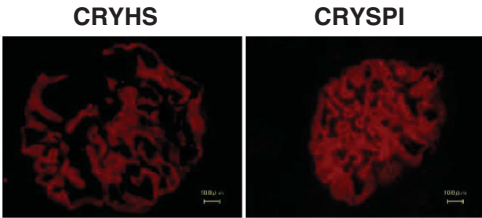

C

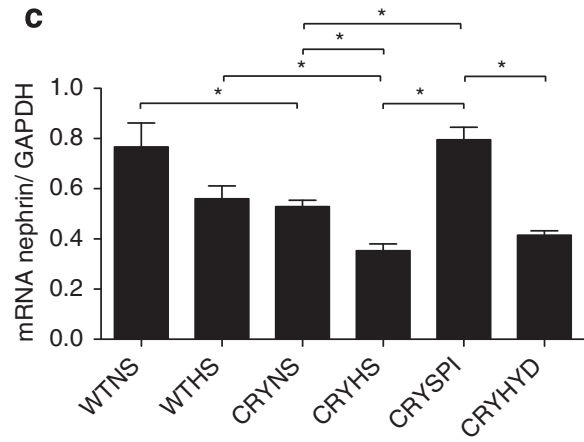

Figure 5 Nephrin expression. (a) A representative image of nephrin immunofluorescent staining and (b) quantification of the signal as the percentage of the glomerulus area $(n=5-10)$. (c) The graphs represent the ratio between mRNA expression and the expression of GAPDH mRNA ( $n=5-7)$. Data are presented as the mean \pm s.e.m. ${ }^{*} P<0.05$ using the Mann-Whitney $U$-test.
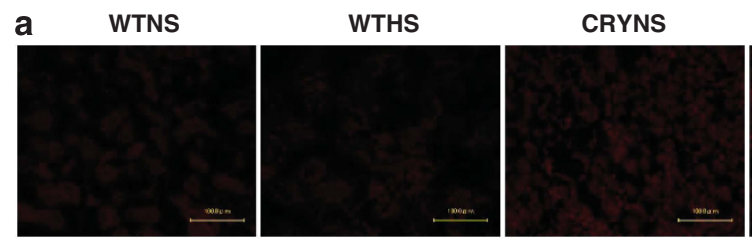

b

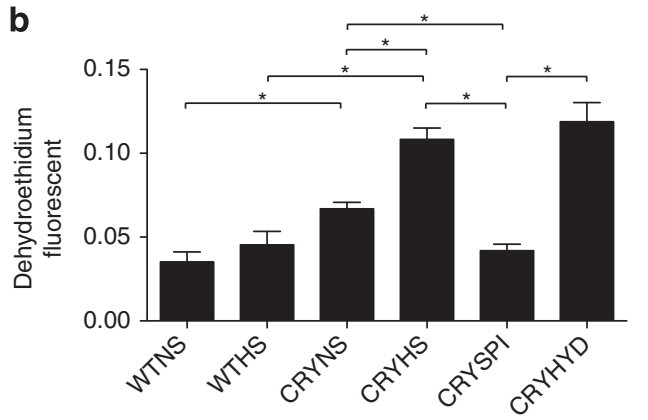

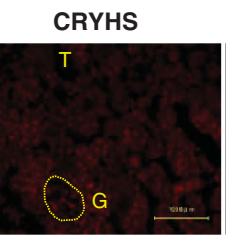

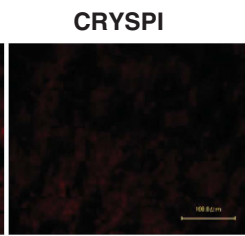

CRYHYD

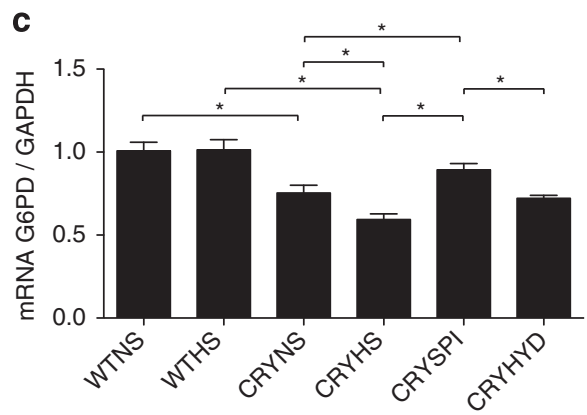

Figure 6 Dihydroethidium (DHE) staining (a), quantification of DHE staining (b) and glucose-6-phosphate dehydrogenase (G6PD) mRNA expression (c). DHE staining quantification was performed in five samples in each group. (a) The DHE-positive staining shows as red fluorescent staining on the cell nucleus on the glomerular (G) and tubular (T) area. (b) Quantification of DHE staining intensity. (c) The graphs represent the ratio between G6PD mRNA expression and the expression of GAPDH mRNA $(n=6-11)$. Data are presented as the mean \pm s.e.m. ${ }^{*} P<0.05$ using the Mann-Whitney $U$-test.

observations, we cannot rule out the fact that chronic endogenous hyperaldosteronism may have induced adaptive mechanisms, which could have limited the deleterious effects of aldosterone. These results may reflect clinical findings in primary aldosteronism patients who often present with microalbuminuria and an increased glomerular filtration rate ${ }^{31}$ that can be successfully treated either by adrenalectomy or MR blocker treatment. ${ }^{32-34}$ Moreover, severe kidney damage is believed to develop only after several years. ${ }^{35}$

In the present study, we confirmed that aldosterone combined with high-salt treatment can induce severe kidney damage. High-salt exposure has been proven to exert pathological effects on the kidney. In rats with renal transplantation, elevated salt intake $(8 \% \mathrm{NaCl})$ induced albuminuria, glomerulosclerosis and tubulointerstitial injury. ${ }^{36}$ In a rat model of metabolic syndrome, high-salt treatment for 4 weeks also promoted hypertension, proteinuria and kidney damage. ${ }^{37}$ In a genetic rat model with lower nephron density that received high-salt treatment for 6 weeks, increased blood pressure and albuminuria were observed. ${ }^{38}$ Interestingly, the pathological effect of salt is related to MR activation. A previous study showed that MR blockers could ameliorate hypertension, proteinuria and kidney injury in unilateral nephrectomized rats receiving 4 weeks of high-salt treatment. ${ }^{39}$ Another study in saltsensitive Dahl rats receiving high-salt treatment indicated the presence of increased blood pressure, albuminuria, kidney damage and impaired MR signaling in the kidney. ${ }^{40}$ Therefore, we suggest that kidney damage in high-salt-treated Cry 1, 2 DKO mice was caused by MR activation owing to both hyperaldosteronism and high-salt exposure. 
High-salt-induced kidney damage in Cry 1, 2 DKO mice was independent of blood pressure. We used low-dose spironolactone (an MR blocker) to inhibit the effect of aldosterone without decreasing blood pressure. One study reported that the administration of spironolactone at a dose of $6-\mathrm{mg} \mathrm{kg}^{-1}$ body weight per day did not decrease blood pressure in an angiotensin II-treated mice model. Moreover, this low dose of spironolactone did not decrease urinary potassium levels as already observed. ${ }^{41}$ Spironolactone treatment decreased the urinary albumin level, nephrin expression and ROS production but did not change the creatinine clearance in the CRYHS group. Increased creatinine clearance in cases of hyperaldosteronism and high-salt exposure, as observed in Cry 1, 2 DKO mice, may result from a functional adaptation induced by increased sodium reabsorption and body fluid volume. This adaptation may lead to hypertension, increased renal perfusion pressure, suppressed renin activity and decreased intrarenal vascular resistance. ${ }^{35}$ In WT mice, the creatinine clearance did not increase because both blood pressure and aldosterone levels remained stable while they were on the highsalt diet. Because the spironolactone dose used in the present study did not decrease the blood pressure in Cry 1, 2 DKO mice, we suggest that renal perfusion and intrarenal vascular resistance did not change either, which would explain the stable creatinine clearance level. To further analyze the effect of aldosterone on kidney damage independent of blood pressure, we administered a direct vasodilator, hydralazine. Hydralazine decreases blood pressure by inducing arterial smooth muscle relaxation. ${ }^{42}$ Therefore, the effect in this group represents a decrease in blood pressure without any interference in the aldosterone-MR interaction. Despite the decrease in blood pressure, treatment with hydralazine in high-salt-treated Cry 1, 2 DKO mice did not improve creatinine clearance, albuminuria, nephrin expression or ROS production. In another study of rats treated with aldosterone, $1 \% \mathrm{NaCl}$ and hydralazine for 4 weeks, a similar result was noted; in the present study, hydralazine treatment decreased blood pressure but did not change the creatinine clearance value, albuminuria, kidney sclerosis or ROS production. ${ }^{11}$ In certain normal, hypertensive and congestive heart failure patients, hydralazine does not appear to influence glomerular filtration rate. ${ }^{43,44}$ The fact that the change in renal plasma flow produced by a vasodilator did not change the glomerular filtration rate indicates the presence of a filtration pressure disequilibrium. ${ }^{43}$ Therefore, given that treatment with spironolactone, but not hydralazine, prevented kidney damage, we suggest that the deleterious effects of salt and hyperaldosteronism on the kidney were specifically due to MR activation.

Considering the role of cryptochromes in the circadian rhythm, the effect of the cryptochrome deletion in Cry 1, 2 DKO mice cannot be disregarded. The increase in urinary albumin levels, decrease in nephrin expression and increase in ROS production in high-salttreated CRYHS mice were inhibited by spironolactone treatment. Thus, we assumed that the kidney damage in the Cry 1, 2 DKO mice was related to hyperaldosteronism.

Finally, the levels of nephrin expression and ROS production in the mice in the spironolactone-treated group were better than those in the CRYNS group and comparable to those in the WTNS group. Thus, in addition to inhibiting the combined effect of hyperaldosteronism and salt, spironolactone may also suppress the effect of hyperaldosteronism alone.

In conclusion, our study demonstrated that the deleterious effects of aldosterone on the kidney might be independent of high-salt exposure and high blood pressure, but high-salt treatment exacerbates these effects. Based on our findings, we suggest that controlling blood pressure may not be sufficient to protect the kidney in primary aldosteronism; however, directly blocking aldosterone may be sufficient to protect the kidney in such cases.

\section{CONFLICT OF INTEREST}

The authors declare no conflict of interest.

\section{ACKNOWLEDGEMENTS}

This study was supported by following grants: The Science Research Promotion Fund from The Promotion and Mutual Aid Corporation for Private Schools of Japan (to NE). Faculty Resources Grant, Kobe University, the Global COE Program, Global Center of Excellence for Education and Research on Signal Transduction Medicine in the Coming Generation from the Ministry of Education, Culture, Sports, Science, and Technology (MEXT) of Japan (to DAAN).

1 Porter GA, Edelman IS. The Action of aldosterone and related corticosteroids on sodium transport across the toad bladder. J Clin Invest 1964; 43: 611-620.

2 Connell JMC, Davies E. The new biology of aldosterone. J Endocrinol. 2005; 186 $1-20$.

3 Remuzzi G, Cattaneo D, Perico N. The aggravating mechanisms of aldosterone on kidney fibrosis. J Am Soc Nephrol 2008; 19: 1459-1462.

4 Fan YY, Kohno H, Hitomi H, Kitada K, Fujisawa Y, Yatabe J, Felder RA, Ohsaki H, Rafiq K, Sherajee SJ, Noma T, NIshiyama A, Nakano D. Aldosterone/mineralocorticoid receptor stimulation induces cellular senescence in the kidney. Endocrinology 2011; 152: $680-688$.

5 Conn JW, Knopf RF, Nesbit RM. Clinical characteristics of primary aldosteronism from an analysis of 145 cases. Am J Surg 1964; 107: 159-172.

6 Greene EL, Kren S, Hotetter TH. Role of aldosterone in the remnant kidney model in the rat. J Clin Invest 1996; 98: 1063-1068.

7 Imazu Y, Muto S, Fujisawa G, Nakazawa E, Okada K, Ishibasi S, Kusano E. Spironolactone suppresses peritubular capillary loss and prevents deoxycorticosterone acetate/salt-induced tubulointerstitial fibrosis. Hypertension 2008; 51: 749-754.

8 Blasi ER, Rocha R, Rudolp AE, Blomme EA, Polly ML, McMahon EG. Aldosterone/salt induces renal inflammation in hypertensive rats. Kidney Int 2003; 63: 1791-1800.

9 Krammer AB, van der Meulen EF, Hamming I, van Goor H, Navis G. Effect of combining ACE inhibition with aldosterone blockade on proteinuria and renal damage in experimental nephrosis. Kidney Int 2007; 71: 417-424.

10 Rocha R, Stier CT Jr, Kifor I, Ochoa-Maya MR, Rennke HG, Williams GH, Adler GK. Aldosterone: a mediator of myocardial necrosis and renal arteriopathy. Endocrinology 2000; 141: 3871-3878.

11 Queisser N, Amann K, Hey V, Habib SL, Schupp N. Blood pressure has only minor influence on aldosterone-induced oxidative stress and DNA damage in vivo. Free Radic Biol Med 2013; 54: 17-25.

12 Klanke B, Cordasic N, Hartner A, Schmieder RE, Veelken R, Hilgers KF. Blood pressure versus direct mineralocorticoid effects on kidney inflammation and fibrosis in DOCAsalt hypertension. Nephrol Dial Transplant 2008; 23: 3456-3463.

13 Brem AS, Morris DJ, Ge Y, Dworkin L, Tolbert E, Gong R. Direct fibrogenic effects of aldosterone on normotensive kidney: an effect modified by 11-HSD activity. Am J Physiol Renal Physiol 2010; 298: F1178-F1187.

14 Sancar A. Regulation of the mammalian circardian clock by cryptochrome. J Biol Chem 2004; 279: 34079-34082.

15 Doi M, Takahashi Y, Komatsu R, Yamazaki F, Yamada H, Haraguchi S, Emoto N, Okuno Y, Tsujimoto G, Kanematsu A, Ogawa O, Todo T, Tsutsui K, van der Horst GT, Okamura $\mathrm{H}$. Salt-sensitive hypertension in circadian clock-deficient Cry-null mice involves dysregulated adrenal Hsd3b6. Nat Med 2010; 16: 67-74.

16 Vitaterna MH, Selby CP, Todo T, Niwa H, Thompson C, Fruechte EM, Hitomo K, Thresher RJ, Ishikawa T, Miyazaki J, Takahashi JS, Sancar A. Differential regulation of mammalian period genes and circardian rhythmicity by cryptochromes 1 and 2. Proc Natl Acad Sci USA 1999; 96: 12114-12119.

17 Fan H, Yang HC, You L, Wang YY, He WJ, Hao CM. The histone deacetylase, SIRT1, contributes to the resistance of young mice to ischemia/reperfusion-induced acute kidney injury. Kidney Int 2013; 83: 404-413.

18 Takagi N, Tanizawa T, Kon V, Fogo AB, Ichikawa I, Ma J. Mineralocorticoid receptor blocker protects against podocyte-dependent glomerulosclerosis. Nephron Extra 2012; 2: $17-26$

19 Funder JW. Mineralocorticoid receptors and cardiovascular damage: it's not just aldosterone. Hypertension 2006; 47: 634-635.

20 Matsunaga M, Hara A, Song TS, Hashimoto M, Tamori S, Ogawa K, Morimoto K, Pak $\mathrm{CH}$, Kawai C, Yoshida O. Asymptomatic normotensive primary aldosteronism: case report. Hypertension 1983; 2: 240-243.

21 Nishimiya T, Kikuchi K, Oimatsu H, Ota S, Nakamura Y, Shimamoto K, limura 0. A case of normotensive primary aldosteronism-comparison with 13 previously experienced cases with hypertension. Endocrinal Jpn 1984; 2: 159-164.

22 Loh KC, Koay ES, Khaw MC, Emmanuel SC, Young WF Jr.. Prevalence of primary aldosteronism among Asian hypertensive patients in Singapore. J Clin Endocrinol Metab 2000; 85: 2854-2859. 
23 Shargorodsky M, Zimlichman R. Primary aldosteronism: the most frequent form of secondary hypertension. Isr Med Assoc J 2002; 4: 32-33.

24 Lombès M, Farman N, Oblin ME, Baulieu EE, Bonvalet JP, Erlanger BF, Gasc JM. Immunohistochemical localization of renal mineralocorticoid receptor by using an antiidiotypic antibody that is an internal image of aldosterone. Proc Natl Acad Sci USA 1990; 87: 1086-1088.

25 Nishiyama A, Yao L, Fan Y, Kyaw M, Kataoka N, Hashimoto K, Nagai Y, Nakamura E, Yoshizumi M, Shokoji T, Kimura S, Kiyomoto H, Tsujioka K, Kohno M, Tamaki T, Kajiya $\mathrm{F}, \mathrm{Abe} \mathrm{Y}$. Involvement of aldosterone and mineralocorticoid receptors in rat mesangial cell proliferation and deformability. Hypertension 2005; 45: 710-716.

26 Shibata S, Nagase M, Yoshida S, Kawachi H, Fujita T. Podocyte as the target for aldosterone: roles of oxidative stress and sgk1. Hypertension 2007; 49: 355-364.

27 Ruotsalainen V, Ljungberg $P$, Wartiovaara J, Lenkkeri U, Kestila M, Jalanko $H$, Holmberg C, Tryggvason K. Nephrin is specifically located at the slit diaphragm of glomerular podocytes. PNAS 1999; 96: 7962-7967.

28 Xu Y, Zhang Z, Hu J, Stillman IE, Leopold JA, Handy DE, Loscalzo J, Stanton RC. Glucose-6-phosphate dehydrogenase-deficient mice have increased renal oxidative stress and increased albuminuria. FASEB J 2010; 24: 609-616.

29 Yoshida K, Kim-Mitsuyama S, Wake R, Izumiya Y, Yukimura T, Ueda M, Yoshiyama M, Iwao $\mathrm{H}$. Excess aldosterone under normal salt diet induces cardiac hypertrophy and infiltration via oxidative stress. Hypertens Res 2005; 28: 447-455.

30 Leopold JA, Dam A, Maron BA, Scribner AW, Liao R, Handy DE, Stanton RC, Loscalzo J. Aldosterone impairs vascular reactivity by decreasing glucose-6-phosphate dehydrogenase activity. Nat Med 2007; 13: 189-197.

31 Sechi LA, Di Fabio A, Bazzocchi M, Uzzau A, Catena C. Intrarenal hemodynamics in primary aldosteronism before and after treatment. J Clin Endocrinol Metab 2009: 1191-1197.

32 Kok KY, Yapp SK. Laparoscopic adrenal sparing surgery for primary hyperaldsteronism due to aldosterone producing adenoma. Surg Endosc 2002; 1: 108-1011.

33 Fourkiotis V, Vonend O, Diederich S, Fischer E, Lang K, Endress S, Beuschlein F, Willenberg HS, Rump LC, Allolio B, Reincke M, Quinkler MMephisto Study Group. Effectiveness of eplerenone or spironolactone treatment in preserving renal function in primary aldosteronism. Eur J Endocrinol 2012; 168: 75-81.
34 Sechi LA, Novello M, Lapenna R, Baroselli S, Nadalini E, Colussi GL, Catena C. Long term renal outcomes in patients with primary aldosteronism. JAMA 2006; 295 : 2638-2645.

35 Catena C, Colussi GL, Sechi AL. Kidney in primary aldosteronism: key determinant of treatment outcome. World J Hypertens 2012; 2: 1-6.

36 Sanders PW, Gibbs CL, Akhi KM, MacMillan-Crow LA, Zinn KR, Chen YF, Young CJ, Thompson JA. Increased dietary salt accelerates chronic allograft nephropathy in rats. Kidney Int 2001; 59: 1149-1157.

37 Nagase M, Matsui H, Shibata S, Gotoda T, Fujita T. Salt-induced nephropathy in obese spontaneously hypertensive rats via paradoxical activation of the mineralocorticoid receptor: role of oxidative stress. Hypertension 2007; 50: 877-883.

38 Kreutz R, Kovacevic L, Schulz A, Rothermund L, Ketteler M, Paul M. Effect of high $\mathrm{NaCl}$ diet on spontaneous hypertension in a genetic rat model with reduced nephron number. J Hypertens 2000; 18: 777-782.

39 Kawarazaki H, Ando K, Shibata S, Muraoka K, Fujita M, Kawarasaki C, Fujita T. Mineralocorticoid receptor-Racl activation and oxidative stress play major roles in salt induced hypertension and kidney injury in prepubertal rats. J Hypertens 2012; 30: 1977-1985.

40 Shibata S, Mu S, Karawazaki H, Muraoka K, Ishizawa K, Yoshida S, Kawarazaki W, Takeuchi M, Ayuzawa N, Miyoshi J, Takai Y, Ishikawa A, Shimosawa T, Ando K, Nagase M, Fujita T. Rac1 GTPase in rodent kidneys is essential for salt-sensitive hypertension via a mineralocorticoid receptor dependent pathway. J Clin Invest 2011; 121: 3233-3243.

41 Luther JM, Luo P, Wang Z, Cohen SE, Kim HS, Fogo AB, Brown NJ. Aldosterone deficiency and mineralocorticoid receptor antagonism prevent angiotensin II- induced cardiac, renal and vascular injury. Kidney Int 2012; 82: 643-651.

42 Ebeiqbe AB, Aloamaka CP. Mechanism of hydralazine-induced relaxation of arterial smooth muscle. Cardiovasc Res 1985; 19: 400-405.

43 Wilkinson EL, Backman $\mathrm{H}$, Hecht HH. Cardiovascular and renal adjustment to hypotensive agents. Circulation 1952; 31: 872-879.

44 Cogen JJ, Humphreys MH, Carlson CJ, Rapaport E. Renal effects of nitroprusside and hydralazine in patients with congestive heart failure. Circulation 1980; 61: 316-323. 